\title{
ELEMENTOS TEÓRICOS EN EL ABORDAJE DE LAS INTERACCIONES COMUNICATIVAS EN LA CLASE
}

\section{THEORETICAL ELEMENTS IN THE APPROACH TO COMMUNICATIVE INTERACTIONS IN THE CLASSROOM}

\author{
Adrián Barrientos Córdoba* \\ Claudio Córdoba Alfaro** \\ Keiner García Calderón***
}

Fecha de recepción: 01/08/2019

Fecha de aceptación: 25/09/2019

Resumen: El presente ensayo de naturaleza académica y argumentativa es un acercamiento a los principales elementos teóricos necesarios para el abordaje de las interacciones comunicativas en el ámbito educativo, entre los cuales se encuentran las interacciones y sus tipos, la comunicación y el lenguaje, el contexto sociocultural, los procesos de enseñanza y aprendizaje y las tradiciones epistemológicas. El estudio de estos elementos teóricos es el marco de referencia que amplía el hecho educativo más allá de la transmisión de contenidos, puesto que busca visualizar el acto educativo desde un plano holístico que permita analizar la educación desde lo que ocurre en el aula.

Palabras claves: educación; comunicación; lenguaje; enseñanza; aprendizaje; aula.

Abstract: The present essay it is an approach of the main theoretical elements necessary for the approach of the communicative interactions in the educational field, among which are; the interactions and their types, communication and language, the sociocultural context, the teaching and learning processes and the epistemological traditions. The study of these theoretical elements is the frame of reference that extends the educational fact beyond the transmission of content, seek to visualize the educational act from a holistic level that allows to analyze education from what happens in the classroom.

Keywords: Education; Communication; Language; Teaching; Learning; Classrooms.

* Costarricense. Licenciado en la Enseñanza de los Estudios Sociales y la Educación Cívica por la Universidad de Costa Rica (UCR), Costa Rica. Docente e investigador independiente. Correo electrónico: adrian.barrientoscordoba@ucr.ac.cr

** Costarricense. Licenciado en la Enseñanza de los Estudios Sociales y la Educación Cívica por la Universidad de Costa Rica (UCR), Costa Rica. Docente e investigador independiente. Correo electrónico: claudio.cordoba@ucr.ac.cr

*** Costarricense. Licenciado en la Enseñanza de los Estudios Sociales y la Educación Cívica por la Universidad de Costa Rica (UCR), Costa Rica. Actualmente labora en programas de educación abierta con el Ministerio de Educación Pública (MEP), Costa Rica. Correo electrónico: keiner.garcia@ucr.ac.cr 


\section{Introducción}

El proceso educativo desde una perspectiva tradicional se ha entendido como la transmisión de contenidos disciplinares por parte del docente hacia el estudiantado, lo cual constituía el acto educativo; sin embargo, desde los nuevos enfoques se comprende que el proceso educativo como tal va más allá de ello; dado que desde las nuevas líneas investigativas en educación se plantea que, para comprender el fenómeno educativo este debe ser analizado de manera integral y holística, debido a que existen otros elementos que forman parte de este y deben ser considerados.

Uno de estos elementos a considerar es el que se refiere a las interacciones comunicativas en educación, tal como plantea Rizo (2007), en ellas, tanto docentes como estudiantes, son sujetos emisores y receptores de información, que generan interpretaciones de sistemas de mensajes, las cuales están enmarcadas dentro de un proceso socializador en el que los actores logran relacionarse y entenderse. A lo cual Herrero (2012) agrega que se encuentran vinculadas con el entorno de la institución educativa y la manera en que los actores construyen sus diálogos en la clase, lo cual es esencial, puesto que no solo se transmiten contenidos disciplinares entre actores educativos, sino que se enlaza directamente con la cotidianidad de lo que sucede en la clase.

Es por lo anterior que el presente ensayo de naturaleza académica y argumentativa pretende exponer un abordaje teórico de los principales elementos que se deben considerar para comprender las interacciones comunicativas y su papel en el proceso educativo, con el fin de que sea un insumo para los docentes en su práctica profesional diaria, en busca de mejorar los procesos de enseñanza y aprendizaje desde lo que ocurre en la clase. Los elementos teóricos por considerar para realizar dicho abordaje son los siguientes: las interacciones comunicativas en educación y sus tipos, el contexto sociocultural, la comunicación y el lenguaje, y los procesos de enseñanza y aprendizaje.

La selección de los elementos teóricos abordados en este artículo responde a la necesidad de visualizar cómo estos convergen en el desarrollo de los procesos de enseñanza y aprendizaje en la clase, para lo cual se vislumbraron dentro de la asignatura de Estudios Sociales, debido al enfoque social de dicha materia, el cual fomenta estos elementos en la clase y los actores que interactúan en esta. Si bien estos elementos forman parte de la dinámica de las clases desarrolladas en cualquier asignatura, el abordaje del tema se hizo desde los Estudios Sociales. 


\section{Interacciones en educación}

El ser humano vive en una constante interacción con lo que lo rodea, tanto con las personas como con su entorno, el cual también influye en su formación; en esa línea, Cerda (2001) plantea que estos procesos de interacción van a tener una fundamental importancia en la actividad educativa, donde tanto docente como estudiante forman parte de este circuito de interacciones. Es por lo anterior que en el aula, entendida como el espacio físico donde se desarrolla la clase, tanto el docente y como los estudiantes, así como el trato existente entre estos actores, configuran la interacción que se desarrolla en el hecho educativo.

La temática de las interacciones en educación se ha configurado según los cambios ocurridos en las estructuras educativas con el pasar de los años, lo que supone que se han replanteado nuevos enfoques educativos y con ello han surgido líneas de investigación, las cuales han tomado relevancia debido a los siguientes planteamientos

“...el primero tiene que ver con la crisis del modelo que establece una relación epistemológica jerárquica y unidireccional entre investigación académica y práctica profesional. El segundo, con la aceptación creciente de los enfoques socioculturales y situados de la cognición, el aprendizaje y la enseñanza. Y el tercero, con la importancia creciente otorgada al contexto del aula" (Coll y Sánchez, 2008, p. 15).

Dado lo anterior, los nuevos enfoques investigativos en educación incursionan en el vínculo entre las interacciones educativas y los enfoques socioculturales, entre estos el contexto, que tiene relación con los procesos de socialización, así como de interacción de los actores educativos. La socialización desarrollada entre las personas en el centro educativo, a partir de procesos educativos, permite establecer relaciones de tipo estudiantes-estudiantes y estudiante-docente en donde se construyen principios sociales dado el ambiente social que caracteriza cada centro educativo.

Dentro de la socialización y los procesos de interacción, la comunicación es la base que permite el intercambio entre los actores educativos; en los modelos tradicionales de enseñanza "...las relaciones entre el profesor y los alumnos se dan en términos puramente informacionales o sea la transmisión unidireccional y unilateral de un conjunto de datos e información" (Cerda, 2001, p. 35), en cambio, desde los modelos actuales de enseñanza se incentiva una comunicación dinámica que promueva la bidireccionalidad y un papel activo de ambos actores.

A partir de la comunicación desarrollada entre docente-estudiante, entre en juego la empatía existente entre estos, dado que ella facilita los vínculos de cercanía o distanciamiento, en donde la "simpatía mutua" permita la 
construcción de un ambiente idóneo que promueva una mejor práctica educativa; sobre esto Molina (1985) expone que se requiere considerar la empatía, dado que, cuando existe una relación empática entre docente y estudiante, la reacción estudiantil responde de diferente manera que con el docente que no le genere empatía, lo que dificulta un adecuado ambiente de clase.

Por su parte, en lo que corresponde a las interacciones entre estudiantes, constituyen parte importante en la producción de nuevas situaciones, planteamiento de tareas, así como formas de solución de problemas; según Coll (1984), este tipo de interacción es preponderante, debido a que influye en la consecución de los objetivos educativos planteados. Sin embargo, no solo influye en el proceso educativo académico, sino también en el tipo de relación que pueda existir entre el estudiantado, la cual, si no es bien atendida, puede generar incluso situaciones conflictivas, que afecten al grupo si estas se descontrolan, o, por otra parte, predeterminar roles dentro del grupo.

Por lo anterior, en este tipo de interacciones "...la conducta y las creencias de los alumnos están condicionadas en gran medida por los pequeños grupos que se forman dentro del aula -tales como las camarillas de amigos-, y los grupos de cohesión que existen dentro de la escuela" (Molina, 1985, p. 105), por lo que las interacciones entre pares están determinadas por la dinámica desarrollada por el mismo grupo.

En ese tópico, Coll (1984) señala que esta relación entre iguales resulta trascendental en el proceso de socialización, dada la adquisición de competencias y destrezas sociales, si se considera que el proceso educativo es una educación para la vida y no solamente enfocada en la adquisición de conocimientos, las interacciones desarrolladas forman parte intrínseca de los procesos de enseñanza y aprendizaje, y por lo tanto deben ser planificadas por el docente, dado que "...no basta con colocar unos alumnos al lado de otros y permitirles que interactúen para obtener automáticamente unos efectos favorables. El elemento decisivo no es la cantidad de la interacción, sino su naturaleza" (Coll, 1984, p. 120).

\section{Tipos de interacciones}

Una vez comprendido el marco en el que se desarrollan las interacciones comunicativas, es preciso señalar los diferentes tipos de interacciones que se encuentran presentes en la acción educativa, así como los actores y elementos presentes en ellas; estas, descritas por Bixio (2002), son: la interacción sociocognitiva, la interacción cognitiva y la interacción contextual. Todas estas se encuentran interrelacionadas entre sí, a pesar de que cada una tiene su especificidad en el análisis del hecho educativo, por lo que resulta imprescindible un abordaje holístico de la temática.

Sin embargo, tradicionalmente en escenarios de investigación educativa, se ha privilegiado el análisis de la interacción de tipo sociocognitiva -docente- 
estudiante-, caracterizada por una preponderancia del papel del cuerpo docente sobre del alumnado; no obstante, la interacción sociocognitiva -estudianteestudiante- es relevante no sólo para comprender procesos educativos, sino también psicológicos, cognitivos o sociales que sirven para interpretar situaciones de aula.

Por otra parte, se encuentran las interacciones cognitivas -estudiantecontenido-, caracterizadas por la relación que existe entre el alumnado y los contenidos de la asignatura respectiva, la cual es el fundamento de los procesos de enseñanza y aprendizaje, puesto que las temáticas de estudio concretas son el punto de partida de la interacción que sucede en el aula.

Por último, las interacciones contextuales -estudiante-estudiante-objeto de conocimiento-docente-contexto- son el tipo de interacción que engloba los elementos de los anteriores tipos de interacciones, este tipo de interacción va más allá del lugar específico donde se desarrolla el acto educativo, sino que trasciende los espacios educativos e involucra el entorno donde se desarrollan el estudiantado en su cotidianidad y la influencia que esta genera en su vida académica.

En general, los tipos de interacciones configuran un entramado que permite estudiar con detalle distintos procesos educativos que surgen de manera cotidiana en las aulas de clase, en donde se involucra al docente, al estudiante, a los contenidos de la asignatura y al contexto, lo cual es importante dado que no exhibe la educación como una actividad que traspasa la sola transmisión de contenidos, sino como una actividad integral, como se muestra en la figura 1.

\section{Figura 1}

Esquema de tipos de interacción

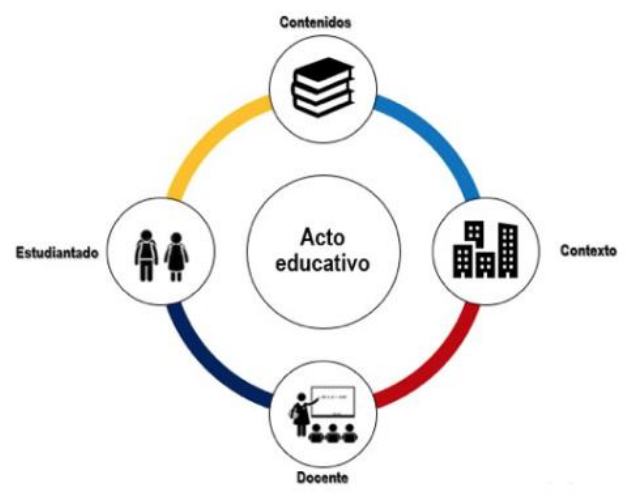

Fuente: elaboración propia.

En esa línea, enfocándose en el aspecto social, las interacciones son un “... tipo de actividad comunicativa realizada por dos o más participantes que se 
influyen mutuamente, en un intercambio de acciones y reacciones verbales y no verbales" (Jauregi, 2012, p. 5), por lo que resalta la participación de los actores educativos involucrados en ella para que estas sean generadas; así mismo, Jauregi (2012) plantea que la existencia del intercambio de estas acciones y reacciones se encuentra inmersa en contextos particulares.

Ahora bien, para que las interacciones se presenten tiene que existir un proceso comunicativo previo, debido a que las interacciones tienen su origen en dicha comunicación que establecen los actores involucrados en el acto educativo. Si el posicionamiento de estos actores es distante entre sí, la comunicación entre ellos se verá limitada; por lo tanto, el docente debe hacer un uso efectivo y eficaz del espacio para que la comunicación entre los actores educativos sea funcional y no se vea restringida.

Las interacciones comunicativas son de interés en temas educativos, ya que estas “...además de estar relacionadas con procesos de socialización, también se vincula la comunicación con entornos educativos, así como a la forma en que los actores que construyen su diálogo" (Herrero, 2012, p. 139). El diálogo en este proceso educativo se encuentra influido en gran medida por el contexto sociocultural, porque a partir de esto se construyen significados o códigos que permiten el entendimiento mutuo.

\section{Comunicación y lenguaje}

El término comunicación es polisémico, es decir, contempla una variedad de definiciones según autores, contextos, necesidades o entornos, o sea, adquiere una concepción multidimensional, puesto que es un concepto que contempla tanto lo funcional como lo semántico (Aguado, 2004). La comunicación presenta dimensiones fundamentales: es relacional, puesto que siempre será un puente de unión entre uno o más elementos diferenciados, también es perceptiva de las diferencias al discriminar lo que se distingue y además posee una estructura particular, puesto que permite la interrelación entre los participantes.

Además, hay elementos que configuran la comunicación: un sujeto emisor y el receptor, los cuales pueden variar su rol de acuerdo al contexto comunicativo, un mensaje que se quiere transmitir construido a partir de códigos de entendimiento mutuo que permitan la comunicación entre los sujetos, un canal que sirve como medio para emitir el mensaje, ya sea verbal o no verbal, y por último, el contexto que determina en gran medida la configuración de los elementos comunicativos, que influyen en los procesos de socialización. Es por lo anterior que cada situación comunicativa se encuentra enmarcada en un conjunto de circunstancias de acuerdo con la forma en la que se relacionan los elementos comunicativos, lo que permite el intercambio comunicativo entre los sujetos (Fajardo, 2009).

El proceso comunicativo se puede generar desde diversos modelos, en donde cada uno de esos elementos es dinámico o existen roles preestablecidos, entre ellos el modelo unidireccional y otro bidireccional, como se muestra en la 
figura 2; sobre esto Kaplún (2002) señala que en educación los modelos más comunes son relacionados con el unidireccional, caracterizado por una situación en donde hay un emisor que envía su mensaje al receptor y que este está asociado a un modelo educativo tradicional al haber roles comunicativos rígidos en la clase, es decir el docente es quien emite mensajes de forma activa y el estudiante solo recibe de forma pasiva. En cuanto al bidireccional, este se fundamenta en una relación comunicativa en la que el rol de emisor y receptor se intercambian constantemente entre actores educativos, lo cual permite un modelo educativo alternativo al tradicional en que se le da participación al estudiante y que este a su vez sea partícipe de la construcción de su conocimiento.

\section{Figura 2}

Esquema modelos comunicativos según Kaplún (2002)

\section{Modelos comunicativos}

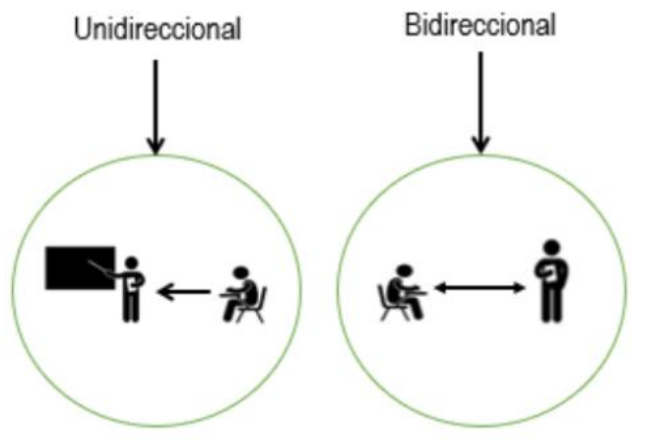

Fuente: elaboración propia.

Luego del abordaje de forma general del tema de comunicación, así como de los elementos comunicativos y modelos más tradicionales en el ámbito educativo, se derivan del lenguaje como la base para generar el proceso comunicativo, por lo que es necesario considerar dicho lenguaje, así como las diferentes formas en la que se expresa.

En relación con lo anterior, el lenguaje funciona como un elemento de interacción que les permite a las personas transmitir o intercambiar mensajes, los cuales tienen diferentes significados según sus contextos sociales. De acuerdo con Amodio (2006), el desarrollo de la capacidad de las personas para percibir su entorno, relacionarse con los demás y producir conocimientos propios se realiza a través del lenguaje -como una herramienta o puente, necesario para conectarse con el mundo externo y con nuestro mundo interior-; con lo anterior es claro que el lenguaje constituye un conector con la percepción propia del individuo en 
relación con lo que lo rodea, lo que le permite interactuar con los demás.

Dentro del lenguaje se derivan dos maneras en las que este se expresa, el primero denominado lenguaje verbal, el cual, según Cruz (2002), ha sido considerado como la base de la comunicación, por motivo de que es el que las personas perciben de manera consciente, lo emplean de forma natural y lo consideran como el medio para transmitir un mensaje, ya sea por sonidos fónicos de las palabras u oraciones que ocurren de forma planificada o de manera espontánea. El segundo, denominado lenguaje no verbal, el cual Landa y Sánchez (2007) expresan que se concibe como el proceso de emitir y recibir mensajes que acompañan, modifican o reemplazan el sonido verbal, sin ser completamente independiente de este último; está vinculado con una serie de expresiones, miradas, gestos y señas que las personas pueden utilizar y percibir de manera consciente o inconsciente.

En educación, Vigotsky (1995) plantea que el lenguaje es el medio por el cual estudiantes y docentes realizan intercambios en la construcción de diálogos, a partir de una temática en donde se desarrolla una expresión interactiva de las ideas por parte de los actores educativos, ya que durante las clases crean una interacción específica por la cual liberan el pensamiento por medio de todos los componentes sensoriales, es decir, mediante los distintos tipos de lenguaje. Vigotsky (1991), a su vez, menciona que dicho lenguaje se encuentra inmerso en un contexto específico, el cual, influenciado por la cultura propia de este, determinará los códigos que regirán dicho lenguaje; por ello, el contexto es uno de los elementos a considerar en el análisis de las interacciones.

\section{Contexto sociocultural}

El ser humano y su desarrollo a lo largo de la historia lo ha llevado a convivir en sociedad, para lo cual ha establecido modelos determinados en las formas de vida para que dicha convivencia se logre; de manera similar, se crea un sistema cultural y educativo que introduce a las personas en la sociedad para que convivan.

En los procesos educativos formales constituye una manera de insertar al sujeto en una cultura particular, la cual podrá tener la capacidad de influir en las formas de comportamiento del ser humano, esta a su vez “...transcurre en medio de un marco social o interpersonal donde estamos en constante interacción con otras personas desde la infancia hasta la vejez y, en general, la vida humana resulta difícil de concebir fuera del marco de estas relaciones sociales" (Cerda, 2001, p. 30), por lo que es intrínseco a la naturaleza del ser humano y va a estar siempre presente en la vida cotidiana de este.

Por otro lado, Rodríguez y Romeu (2010) consideran que, en ámbitos educativos y su relación con el contexto, la cultura tiene un rol preponderante en la cosmovisión de las personas, y esta configura al individuo en las relaciones que establece con las personas y su entorno. Vigotsky (1991), en su estudio sobre la niñez y la cultura, plantea que en su desarrollo el niño, no solo se apropia del 
contenido de la experiencia cultural, sino que, además, adquiere las formas y procedimientos de la conducta cultural; siendo los centros educativos uno de los espacios donde esto ocurre.

Sin embargo, la institución educativa en sí misma no es la que reproduce la cultura, sino que va acompañada por la influencia que la comunidad tiene en ese proceso, así como el papel de la familia como la base inicial de socialización de la persona. En esa línea, Motta (2010) establece la existencia de un vínculo entre centro educativo y comunidad, dado que no se presentan de manera aislada, en donde la familia está inscrita en dicha comunidad, es por lo anterior que "...el centro educativo debe ejercer un fuerte liderazgo en la comunidad basado en acciones educativas permanentes orientadas a la solución de problemas y al mejoramiento de la calidad de vida de su población" (Motta, 2010, p. 240); por lo tanto, el centro educativo, la comunidad y la familia son los principales espacios de formación cultural dentro de una sociedad.

\section{Figura 3}

Esquema de relación contexto-comunidad-familia

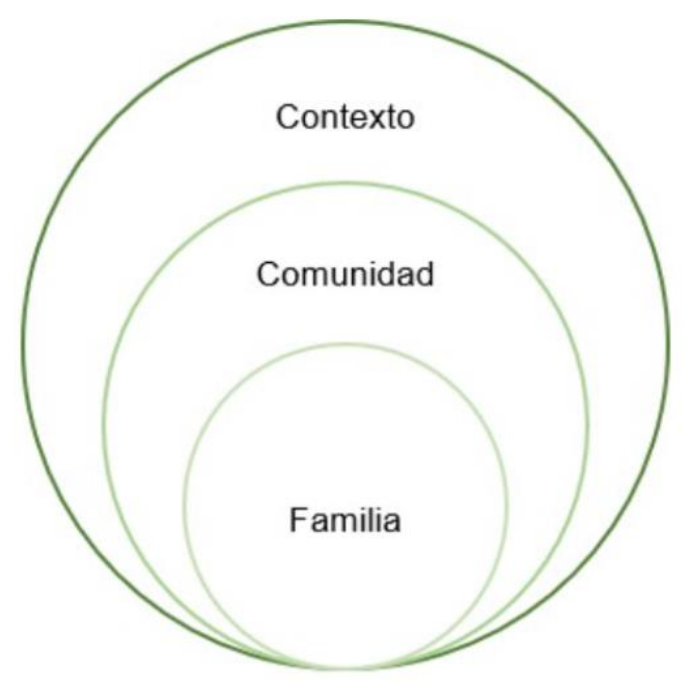

Fuente: elaboración propia.

Con base en la figura 3, la correlación de estos tres elementos permite facilitar los procesos comunicativos entre estos espacios para la búsqueda de soluciones de las problemáticas sociales, donde la interacción aporta no solo en el proceso pedagógico, sino, además, en lo social. Es por lo anterior que es pertinente que, dentro de la planificación, el docente tome en cuenta que estos espacios configuran las interacciones comunicativas que se desarrollan en la clase y que existe una relación recíproca con el contexto sociocultural del que provienen, con el fin de que la socialización y la mediación didáctica de contenidos estén estrechamente ligadas a su realidad. 


\section{Procesos de enseñanza y aprendizaje}

En relación con la mediación didáctica y las interacciones comunicativas, en el hecho educativo se desarrollan los procesos de enseñanza y aprendizaje, los cuales, según una perspectiva tradicional, han sido visualizados de forma aislada uno de otro, es decir, la enseñanza en función del docente, mientras que el aprendizaje es algo asignado solo al estudiantado. Al respecto, Bravo y Cáceres (2006) mencionan que "...en la mayoría de los salones de clase los estudiantes tienen que atender permanentemente al profesor, quien es poseedor de los conocimientos, los transmite como verdades acabadas, dando poco margen para que el estudiante reflexione y llegue a soluciones, de forma independiente" (p. 1). Sumado a esto, el aislamiento se ve reforzado a partir de la fragmentación de los conocimientos y saberes de las diferentes disciplinas que conforman el currículo oficial.

Es por lo anterior que los nuevos enfoques educativos pretenden concebir los procesos de enseñanza y aprendizaje de manera integral sin estar aislados ni adscritos a un determinado actor educativo únicamente, sino que más bien tanto el docente como los estudiantes enseñan y aprenden en la clase; para que se logre tal situación, es necesaria la comunicación a partir de un modelo bidireccional, en el cual se desarrollan interacciones comunicativas. En esa línea, Chanto y Durán (2014) plantean que:

"El paradigma humanista en la educación refleja el interés del ser humano por superar vacíos de la educación tradicional u otras ideologías han dejado en el ser. Por ello, el reconocimiento del potencial y las cualidades individuales representan una necesidad que debe ser abarcada y atacada por el sector educativo, con miras a brindar un mejor apoyo a la formación y consolidación pedagógica en nuestras sociedades" (p.25).

En ese tópico, Meneses (2007) manifiesta que "la enseñanza no puede entenderse más que en relación al aprendizaje; y esta realidad relaciona no sólo a los procesos vinculados a enseñar, sino también a aquellos vinculados a aprender" (p. 32); por ende, el abordaje de estos dos conceptos debe realizarse de manera holística, es decir, que el docente cuando enseña puede creer en su concepción que, por añadidura, el estudiante estará aprendiendo sin comprobar que este acto esté ocurriendo, por lo que en su labor debe evaluar que haya aprendizaje y no solo una transmisión de contenidos.

En esta misma línea, el contexto, así como la realidad social, se encuentran ligados con los procesos de enseñanza y aprendizaje a partir del intercambio comunicativo y de la interacción que surgen entre el estudiantado y el cuerpo docente; es importante señalar que van más allá de un aspecto meramente académico, debido a que "el proceso de enseñanza aprendizaje tiene como propósito esencial favorecer la formación integral de la personalidad del 
educando, constituyendo una vía principal para la obtención de conocimientos, patrones de conducta, valores, procedimientos y estrategias de aprendizaje" (Campos y Moya, 2011, p. 2). Es por ello que la educación no es únicamente la transmisión de contenidos oficiales o académicos, sino que también se enseñan y aprenden aspectos de diversa índole que permiten formar al estudiantado como personas desde una perspectiva integral.

Sumado a lo anterior, para el desarrollo de los procesos de enseñanza y aprendizaje, la comunicación es la base, esto porque el acto educativo parte de la comunicación como el medio para facilitar la socialización de los contenidos, en donde el lenguaje permite concretarlo; tal como señala Vigotsky (1995), el lenguaje influye en las capacidades cognitivas de las personas lo que en gran medida determina el desarrollo de los pensamientos y, por lo tanto, la forma que adquieren los nuevos conocimientos.

Por lo tanto, en el abordaje de los procesos de enseñanza y aprendizaje, estos deben ser comprendidos como apartados en constante vinculación, distanciándose de perspectivas que los aíslan, por lo que la comunicación que ocurra en la clase debe ser una herramienta útil para que los actores educativos interactúen de manera que les permita que puedan tanto aprender cómoenseñar.

\section{Tradiciones epistemológicas}

A partir de los procesos de enseñanza y aprendizaje es que surge el hecho educativo, acompañado de la planificación y la mediación que el docente realiza en su práctica profesional, lo que propicia el desarrollo de interacciones de diverso tipo; estas interacciones se encuentran vinculadas en gran medida al modelo epistemológico que el docente emplee en la clase y con el cual se posicione, lo anterior influirá en el tipo de interacción comunicativa que se desarrolle en el aula.

Dentro de las expectativas del docente en su planificación y mediación de la clase, se puede establecer “...como propósito esencial favorecer la formación integral de la personalidad del educando, constituyendo una vía principal para la obtención de conocimientos, patrones de conducta, valores, procedimientos y estrategias de aprendizaje" (Campos y Moya, 2011, p. 2); es por lo anterior que el aula se convierte en un espacio en donde se procura desarrollar actividades y acciones en miras hacia lograr el aprendizaje por parte del estudiantado, lo cual responde al posicionamiento teórico-epistemológico del docente.

Según la tradición epistemológica utilizada, determinará las relaciones que se establecen en la clase por parte de los actores educativos, así como las interacciones comunicativas que entre estos desarrollen. Es por ello que el estudio de las tradiciones epistemológicas es un eje transversal en el estudio de las interacciones comunicativas, a pesar de la existencia de diferentes tradiciones epistemológicas, como por ejemplo las abordadas por Barrón (2015), Linceras y Romero (2016) y Benejam y Pagés (1997); el presente artículo se enfocará en la 
tradición positivista, la humanística y la crítica, debido a que en estas tres se tipifican los roles y funciones que, tanto docente como estudiantes, desarrollan en la clase, lo que permite un abordaje íntegro de cómo las interacciones comunicativas se encuentran presentes en la dinámica de la clase.

En primer lugar, se encuentra la tradición positivista, caracterizada por abordar lo que sucede en la clase como un espacio donde el docente asume una posición central, puesto que posee el conocimiento que se considera válido, mientras que el alumnado debe aprender únicamente, como un sujeto pasivo con una mente vacía que debe ser rellenada con conocimientos objetivos preestablecidos por un currículo oficial (Liceras y Romero, 2016). En cuanto a la metodología utilizada por el docente, se privilegia el obtener resultados a partir de la acumulación de conocimiento, excluyendo posibilidades de creatividad, crítica y participación del estudiantado.

Este tipo de tradición epistemológica, dada su naturaleza, limita la posibilidad de que se desarrolle una interacción comunicativa entre todos los participantes presentes en el aula, debido a que la comunicación la acapara el docente de forma unidireccional hacia el estudiantado en la transmisión de contenidos, limitándose la interacción que se pueda desarrollar entre el grupo de estudiantes.

En segundo lugar, se encuentra la tradición de tipo humanista, esta, según Benejam y Pagés (1997), se relaciona con la noción de que el estudiante es el centro del hecho educativo y, por ende, es este el que debe construir su conocimiento, con el docente como guía que lo motive a que pueda, a partir de descubrir e investigar realizar tal función, con una mediación didáctica que posibilite el crecimiento personal y académico de cada estudiante.

La tradición humanista, desde su planteamiento, evidencia que las interacciones entre los actores educativos son un eje esencial para que se logren los procesos de enseñanza y aprendizaje, con miras hacia un profesorado con cualidades empáticas que permita acercarse al estudiantado y que, a su vez, les posibilite a los estudiantes que interactúen entre sí mediante un modelo comunicativo bidireccional.

En tercer lugar, se encuentra la tradición crítica según Benejam y Pagés (1997), es la tradición en la cual el docente funge como un actor que critica la realidad en la que se encuentra inserto el centro educativo y busca la transformación de esta; por lo que busca que el estudiantado esté orientado hacia la conciencia de la existencia de un sistema de valores que rige su entorno. La mediación didáctica que el docente utiliza permite abordar la reflexión como punto de partida para la crítica de la realidad en la que se encuentran inmersos los actores educativos, en evidencia de problemáticas de diverso tipo.

En esta tradición los actores educativos tienen un papel propositivo y sustentado en una igualdad de condiciones en las interacciones comunicativas que se desarrollan, puesto que este modelo busca deconstruir el sistema jerárquico en el cual la educación se ha construido y promovido tradicionalmente. Además, debido a su visión didáctica, el modelo comunicativo bidireccional es su 
base, puesto que, tanto docente como estudiantes, deben criticar la realidad social en la que se encuentran.

Por último, Mendióroz (2013) señala que las tradiciones epistemológicas permiten comprender diferentes modelos educativos a partir de apartados como los objetivos, la mediación didáctica, el abordaje del conocimiento, la construcción de los procesos de enseñanza y aprendizaje, así como de la forma en la que se desarrolla la clase y las interacciones comunicativas entre los actores educativos.

Por tanto, si se asume que la metodología y las actividades limitan o promueven las interacciones comunicativas según la tradición epistemológica se debería optar, a modo de recomendación, por la postura humanística o crítica, en la cual se promueven interacciones comunicativas al existir mayor facilidad, dado que el estudiantado tiene un papel activo en la clase en el que se fomenta un mayor diálogo entre los actores educativos y su relación con los contenidos.

\section{Conclusiones}

En el estudio de las interacciones comunicativas resulta necesario el abordaje de los elementos teóricos que las conforman, puesto que son el sustento de estas y permiten comprender cómo se configuran las relaciones entre los actores educativos, así como de su importancia en el acto educativo. Lo anterior resulta relevante puesto que permite visualizar las interacciones comunicativas como un objeto de estudio que debe ser tomado en cuenta tanto en la práctica profesional de los docentes como en investigaciones educativas, con miras hacia una mejor comprensión de la realidad educativa en la que se encuentran inmersos los actores educativos.

En primera instancia, se encuentran las interacciones y su relación con la educación, las cuales están adscritas en un entorno sociocultural específico, el cual determinará una serie de conductas, patrones y códigos que influyen en la forma en cómo, tanto docente como estudiantes, se relacionarán en el aula y, por ende, cómo se desarrollará su interacción; las interacciones a su vez se encuentran mediadas por procesos comunicativos entre los actores educativos y pueden ser categorizadas para su mejor comprensión en interacciones cognitivas sociocognitivas, así como interacciones contextuales, las cuales están interrelacionadas entre sí, por lo que deben ser abordadas con el fin de comprender la educación de manera integral.

Ahora bien, en la generación de las interacciones comunicativas, la empatía constituye un aspecto potenciador que posibilita la relación entre los actores, por lo que en la clase debe existir un ambiente de convivencia que promueva confianza y respeto, con el fin de desarrollar cuestiones tanto personales como académicas.

En segunda instancia, como un aspecto fundamental en las interacciones comunicativas se encuentra el lenguaje como la base para la generación de estas, sin embargo, se debe tener presente la existencia de modelos comunicativos en 
educación que influyen en el intercambio de mensajes y que caracterizan en gran medida el papel que cumplen el docente y los estudiantes en la clase; el modelo unidireccional refleja una visión tradicional, puesto que asigna un rol activo o preponderante al docente, relegando al estudiantado, mientras que la alternativa de un modelo bidireccional promueve que los actores educativos compartan los roles de emisor y receptor, lo cual resulta favorable para la construcción del conocimiento colectivo, así como un entendimiento mutuo de ideas.

En tercera instancia, se encuentra el contexto sociocultural, el cual es un factor que integra diversos espacios y cómo estos influyen en las interacciones comunicativas, entre ellas se encuentran la propia institución educativa, la comunidad a la que está adscrita, la familia como el núcleo inicial de socialización y formadora de valores, así como todos los espacios en los que el estudiantado y docente socializan, aportan en la forma en cómo cada persona se relaciona con otra, dado que es a partir de esas experiencias que moldean los procesos de interacción y con estos el desenvolvimiento y desempeño que cada estudiante tendrá en su proceso educativo. De ello que el aula, así como el desarrollo de la clase, son los espacios donde convergen estos procesos de socialización.

Como cuarta instancia, se encuentran los procesos de enseñanza y aprendizaje, los cuales, enfoques contemporáneos, permiten comprenderlos de manera conjunta y asociados a la interacción comunicativa que se pueda desarrollar entre los actores educativos, puesto que la práctica del docente no supone en sí aprendizaje, el proceso se integra como tal cuando la enseñanza está ligada a la forma en cómo aprende el estudiantado.

En quinta instancia, las tradiciones epistemológicas son significativas, porque según la postura que asuma el docente en relación con la mediación didáctica que utilice en la clase va a influenciar en gran medida las interacciones comunicativas que se desarrollen, puesto que tanto el docente como los estudiantes van a asumir roles o papeles activos o pasivos, lo cual no solo afecta su convivencia, sino también la construcción del conocimiento en el hecho educativo.

Para finalizar, el estudio de las interacciones comunicativas en educación debe considerar los elementos teóricos anteriormente desarrollados, con el fin de que se aborden de manera conjunta y sean de utilidad para el docente; a partir de este abordaje teórico se recomienda implementar la investigación como forma para comprender la cotidianidad de la clase y cómo se configuran las interacciones comunicativas en las que se desenvuelven los actores educativos.

\section{Referencias bibliográficas}

Aguado, J. (2004). Introducción a las teorías de la comunicación y la información. Universidad de Murcia.

Amodio, E. (2006). Cultura, comunicación y lenguajes. Caracas: IESALC 


\section{UNESCO.}

Barrón, C. (2015). “Concepciones y práctica docente. Una revisión”. Revista de Docencia Universitaria, 13(1), 35-56. Recuperado de http://redu.net/redu/files/journals/1/articles/899/public/899-3923-1-PB.pdf

Benejam, P. y Pages, J. (1997). Enseñar y aprender ciencias sociales, geografía e historia en la educación secundaria. Barcelona: Horsori.

Bixio, C. (2002). Enseñar a aprender. Santa Fe: Editorial Homo Sapiens.

Bravo, G. y Cáceres, M. (2006). "El proceso de enseñanza-aprendizaje desde una perspectiva comunicativa". Revista Iberoamericana de Educación, 38(7), 17.

Campos, P. y Moya, R. (2011). "La formación del profesional desde una concepción personalizada del proceso de aprendizaje". Cuaderno de Educación y Desarrollo, 3(28), 1-6. Recuperado de: http://www.eumed.net/rev/ced/28/cpmr.htm

Cerda, H. (2001). El proyecto de aula; el aula como un sistema de investigación y construcción de conocimientos. Bogotá: Magisterio.

Chanto, C. y Durán, M. (2014). "Humanismo educativo en la sociedad del conocimiento". Revista Nuevo Humanismo, 2(1), 25-36.

Coll, C. (1984). "Estructura grupal, interacción entre alumnos y aprendizaje escolar". Revista Infancia y Aprendizaje, 27(28), 119-138. Recuperado de https://aula.infd.edu.ar/aula/archivos/repositorio/500/703/Coll_Estructur a_grupal.pdf

Coll, C. y Sánchez, E. (2008). "Presentación. El análisis de la interacción alumno-profesor: Líneas de investigación”. Revista de Educación, 346(1), 15-32. Recuperado de http://www.revistaeducacion.mepsyd.es/re346/re346_01.pdf

Cruz, I. (2002). Interacciones comunicativas en el salón clases. Estudio de caso del grupo de la escuela primaria Instituto Kiel ciclo escolar 2000-2001 (Tesis pregrado). Universidad Nacional Autónoma de México, México, D.F., México. Recuperado de http://132.248.9.195/ppt2002/0303707/Index.html

Fajardo, U. (2009). "A propósito de la comunicación verbal”. Revista Forma y Función, 22(2), 121-142. Recuperado de https://revistas.unal.edu.co/index.php/formayfuncion/article/view/23763/360 
61

Herrero, P. (2012). "La interacción comunicativa en el proceso de enseñanzaaprendizaje”. Revista electrónica de investigación, 1, 138-143. Recuperado de http:/www.ugr.es/ miguelgr/ReiDoCrea-Vol.1-Art.19Herrero.pdf

Jauregi, K. (2012). La interacción, elemento clave en el proceso de aprendizaje de ELE. XXIII Congreso Internacional de la ASELE, Plurilingüismo y Enseñanza de ELE en Contextos Multiculturales. Girona, España.

Kaplún, M. (2002). Una pedagogía de la comunicación (el comunicador popular). La Habana: Editorial Caminos.

Landa, M. y Sánchez, J. (2007). Estrategias extraverbales en la interacción comunicativa: la interculturalidad en el aula. Las destrezas orales en la enseñanza del español L2-LE. XVII Congreso Internacional de la Asociación del Español como Lengua Extranjera (ASELE) (pp. 721-736). Recuperado de http://cvc.cervantes.es/ensenanza/biblioteca_ele/asele/pdf/17/17_0721.pdf

Liceras, A. y Romero, G. (2016). Didáctica de las Ciencias Sociales. Madrid: Pirámide.

Mendióroz, A. (2013). Didáctica de las ciencias sociales. Universidad de Navarra, Pamplona.

Meneses, G. (2007). "El proceso de enseñanza-aprendizaje: El acto didáctico". Revista de Medios y Educación, 29, 31-65.

Molina, A. (1985). Diálogo e interacción en el proceso pedagógico. Ciudad de México: El Caballito.

Motta, C. (2010). Relación centro educativo, familia y comunidad: Estrategia clave para mejorar la calidad de la educación. San José: EUNED.

Rizo, M. (2007). "Interacción y comunicación en entornos educativos: Reflexiones teóricas, conceptuales y metodológicas". Revista da Associação Nacional dos Programas de Pós-Graduação em Comunicação, 2(16), 1-16. Recuperado de http://www.compos.org.br/seer/index.php/ecompos/article/viewFile/143/144

Rodríguez, B. y Romeu, N. (2010). "Familia-escuela-comunidad: pilares para la inclusión". Revista Interuniversitaria de Formación de Profesorado, 
Universidad de Zaragoza, 24(3), 47-57. Recuperado de http://www.redalyc.org/pdf/274/27419173004.pdf

Vigotsky, L. (1991). Acerca del desarrollo cultural del niño. Moscú: Universidad de Moscú.

Vigotsky, L. (1978). Mind in Society: The Development of Higher Psychological Processes. London: Harvard University Press.

Vigotski, L. (1995). Pensamiento y lenguaje. Teoría del desarrollo cultural de las funciones psíquicas. Buenos Aires: Ediciones Fausto. 